Artigos

\author{
ENCARTE DIGITAL
}

\title{
Cinco dimensiones del cosmopolitismo
}

\author{
Five dimensions of cosmopolitism
}

Daniel Loewe*

\begin{abstract}
Resúmen: El artículo examina cinco dimensiones de la ciudadanía cosmopolita: el derecho democrático cosmopolita; la justicia distributiva global; las circunscripciones territoriales variables; la sociedad civil global; y la educación cosmopolita, y discute las consecuencias normativas que se siguen de esos entendimientos. De acuerdo al artículo, la ciudadanía cosmopolita es un objetivo político coherente y correcto.
\end{abstract}

Palabras-claves: Cosmopolitismo. Liberalismo. Democracia global. Justicia distributiva. Educación cosmopolita.

\begin{abstract}
The article examines five dimensions of cosmopolitan citizenship: the cosmopolitan democratic law; global distributive justice; variables territorial constituencies; global civil society; and cosmopolitan education. It also discusses the normative implications that follow from these understandings. According to the article, cosmopolitan citizenship is a coherent and correct political objective.
\end{abstract}

Keywords: Cosmopolitism. Liberalism. Global democracy. Distributive justice. Cosmopolitan education

En torno al concepto de "ciudadanía" se pueden organizar múltiples debates de la filosofía política actual. Ya en los noventa "ciudadanía" había llegado a ser una palabra de moda para los defensores múltiples líneas de pensamiento político (Heater 1999; Kymlicka y Norman, 1994). Esta tendencia puede ser retrotraída a diferentes causas, sobre todo a acontecimientos sociales y políticos en cuyo centro está el carácter, la legitimidad y la capacidad existencial del estado (nacional). A menudo, se cuestiona concepciones tradicionales y unitarias de la ciudadanía. Este es el caso de tres conocidos

\footnotetext{
* Doctor en Filosofía por la Universidad de Tübingen (Alemania), profesor de filosofía política de la Escuela de Gobierno de la Universidad Adolfo Ibáñez en Santiago de Chile, investigador del Núcleo Milenio Modelos de Crisis (NS130017)<daniel.loewe@uai.cl>.
}

Civitas, Porto Alegre, v. 15, n. 4, e155-e173, out.-dez. 2015 
debates: multiculturalismo, virtudes ciudadanas, y cosmopolitismo. Este artículo se enfoca en diferentes dimensiones de la ciudadanía cosmopolita y las consecuencias normativas que se siguen de esos entendimientos. Procedo en cinco pasos. Primero me refiero a los tres debates mencionados. Luego, a dos modos de entender el cosmopolitismo (institucional y moral). En tercer lugar indago cinco dimensiones de la ciudadanía cosmopolita. Luego me refiero de un modo conciso a la postulada incompatibilidad entre el cosmopolitismo y las obligaciones ciudadanas. Finalmente, obtengo algunas conclusiones.

\section{Los debates sobre la ciudadanía}

Los debates sobre el multiculturalismo y los derechos de minorías refieren a discusiones sobre los derechos y el estatus de las minorías en sociedades multiétnicas o multinacionales. Ellos se ocupan de temas disímiles que van desde la integración en sociedades plurales democráticas, por sobre planes de estudio multiculturales en escuelas y universidades, nacionalismo, hasta la partición de estados. Característico de estos debates es que la ciudadanía se ve sometida a una presión interior: diferentes grupos dentro de la sociedad políticamente organizada plantean exigencias que, en lenguaje de la ciudadanía, se expresan como: "ciudadanía diferenciada" (Young, 1990), "ciudadanía parcial" (Spinner-Halev, 1994) o "ciudadanía multicultural" (Kymlicka, 1995).

Los debates sobre las virtudes del ciudadano refieren a discusiones sobre las virtudes, prácticas y responsabilidades que caracterizan al ciudadano democrático. Aquí surgen preguntas acerca del mantenimiento y la estabilidad de sociedades liberales democráticas y acerca de la postulada necesidad de un ciudadano activo, cuyas prácticas sobrepasen las exigibles por una teoría liberal. Se contrapone la ciudadanía "activa" a la "pasiva" o "privada", o la republicana a la liberal (Macedo, 1990; Galston, 1991; Dagger, 1997; Höffe, 1999).

Por su parte, los debates sobre el cosmopolitismo refieren a un conglomerado de temas disímiles que, en general y de un modo simplificado, resaltan la necesidad práctica y/o demanda moral de establecer obligaciones normativas e instituciones cuyo alcance vaya más allá de aquel delimitado por las fronteras jurisdiccionales estatales. Corrientemente a la base de estos debates yace la constatación de una supuesta tensión en las teorías liberales entre una fundación universal de principios de justicia y su implementación estado-céntrica. Las discusiones refieren tanto a ámbitos temáticos morales como institucionales, ocupándose de temas tan variados y amplios como "intervención y soberanía", "justicia global" (condiciones constitutivas justas de los mercados, transferencia de recursos etc.), "sistemas educativos 
cosmopolitas", "estructuras supranacionales" o "jurisprudencia internacional" (universalidad de los derechos humanos, prosecución de crímenes de lesa humanidad etc.). Característico de estos casos es que se considera que la ciudadanía es desafiada desde afuera por estructuras supra-estatales o interestatales. En estos contextos se refiere a "ciudadanía múltiple" (Heater, 1999), "ciudadanía postnacional” (Soysal 1997, p. 21), "ciudadanía cosmopolita" (Hutchings y Dannreuther, 1999), "ciudadanía transnacional" (Bauböck, 1994), también a "ciudadanía del mundo" o "ciudadano del mundo" (Nussbaum, 1996; 1997; 2006; Höffe, 1999; 2004), "ciudadanía global" (Turner, 1990), "estatus de ciudadano del mundo" (Habermas, 1992, p. 660; Beck; 1997) y "ciudadanía fragmentada" (Wiener, 1997).

Estos debates no son compartimentos estancos, sino que se vinculan entre sí. Ejemplarmente, muchos promotores del multiculturalismo coinciden con los defensores del cosmopolitismo en que las teorías liberales tradicionales no disponen de ninguna teoría seria sobre las fronteras y su estatus normativo; esto es, una teoría sobre la legitimidad de un trato diferenciado a causa de una contingencia histórica -a que lado de la frontera nacemos. Pero las dos corrientes teóricas intentan superar este manco con estrategias completamente diferentes. Otra vinculación refiere a una supuesta tensión entre las virtudes ciudadanas y la ciudadanía cosmopolita: ya que comúnmente la apelación a las virtudes ciudadanas se lleva a cabo dentro de una concepción paradigmática de ciudadanía unitaria, cuyas fronteras calzan con las fronteras del estado nacional, se suele sospechar que hay una tensión, en ocasiones considerada irresoluble, entre las demandas asociadas con la ciudadanía cosmopolita y las prácticas, virtudes, responsabilidad y lealtad de los ciudadanos. Así se plantea la pregunta acerca de si puede sostenerse en el tiempo una democracia liberal, cuyos miembros se entienden a sí mismos como ciudadanos del mundo.

\section{La perspectiva institucional y la perspectiva moral}

Cosmopolitismo es probablemente el credo silenciosamente compartido por muchas personas activas en organizaciones y agencias internacionales que tratan de avanzar la causa de la implementación global de los derechos humanos (Amnesty International, Oxfam, diferentes agencias de las Naciones Unidas etc.). Parcialmente, se lo comparte también entre aquellos que son activos en organizaciones que buscan avanzar causas medioambientales. Aunque es una posición a favor de la cual en los últimos años se ha argumentado fuertemente (Caney, 2001; 2005; 2007; Fabre, 2005; Moellendorf, 2002; Nussbaum, 2006; Tan, 2000; 2004; 2012), son pocos los que la asumen como punto de partida en la filosofía política. 
La literatura es variada. Lo son tanto los temas que se discuten, como los marcos teóricos en que se articulan las estrategias argumentativas. Hay marcos teóricos contractualistas(Pogge, 1989; 1992; Held, 1995; Beitz, 1999; Barry, 1974; Tan, 2004; 2012) que refieren en mayor o menor grado al modelo rawlsiano (1971), pero que obtienen consecuencias completamente diferentes a aquellas que Rawls considera como correctas (Rawls, 1993; 1999; cf. Loewe, 2015). Marcos teóricos utilitaristas (por ejemplo, Singer, 1972; 2002; 2004; Jones, 1999). Así como teorías cercanas al derecho natural (Nussbaum, 2006; Risse, 2012).

Para el objetivo de este artículo resulta productivo evidenciar algunas de las tesis cosmopolitas centrales. Una primera diferenciación es importante: autores cosmopolitas tienen tanto intereses morales como intereses institucionales (cf. Pogge, 1992, 2002; Beitz, 1999); se distingue entre "moral cosmopolitanism" y "institutional cosmopolitanism". Para una crítica compare O'Neill (2000). Esta diferenciación puede parecer demasiado drástica. Después de todo, una perspectiva cosmopolita moral probablemente nos lleva a apoyar la creación de nuevas instituciones. Sin embargo, ella es apropiada: aunque corrientemente posiciones morales traen consigo consecuencias al nivel institucional, y aunque posiciones institucionales intentan obtener justificaciones apelando a principios morales, una posición moral no tiene que ser caracterizada mediante instituciones (ellas no son una condición necesaria), mientras que una posición institucional no tiene que ser caracterizada mediante su justificación moral.

A la perspectiva institucional podemos llegar mediante argumentos empíricos muy populares. La tesis central puede ser expresada del siguiente modo: en el mundo actual la interacción, coordinación y cooperación de los diferentes actores está tan estrechamente articulada, que a los actores singulares les falta capacidad de acción (cf. Höffe, 1999). El fenómeno de la globalización se presenta como un proceso gradual, pero continuado, económico, social y cultural, que responde a diferentes actores (firmas, agrupaciones de individuos, organizaciones internacionales, estados). Debido al sistema de interacciones estrechamente articuladas surge una necesidad de acción que exige nuevas estructuras institucionales. Por una parte, los estados como actores internacionales tradicionales no pueden implementar ciertos programas y políticas públicas cuando, por ejemplo, las empresas pueden traspasar fronteras. Por otra parte, hay problemas cuya solución exige la coordinación de actores múltiples, como peligrosos desarrollos medioambientales, sociales (por ejemplo, migración) o de seguridad (por ejemplo, terrorismo) que sólo pueden ser manejados mediante mecanismos de coordinación complejos. 
Se puede tener más o menos dudas, por cierto justificadas, acerca de la extensión y valoración de las externalidades, o acerca de la supuesta incapacidad de acción de los estados. No me ocuparé de aquello. Sólo quiero llamar la atención a lo siguiente: si entendemos la ciudadanía como un mecanismo de autodeterminación, como es entendida en la tradición del ciudadano activo de la polis, de la ciudad-estado, y también del estado-nacional moderno, y la necesidad de acción puede ser superada mediante estructuras que se sitúan por sobre las estructuras del estado-nacional, entonces la ciudadanía que ahora necesitaríamos debiese ser localizada a un nivel más alto. Si se trata de un nivel regional o global, depende de los problemas encarados. Con este argumento sólo se puede alcanzar una ciudadanía activa, como autodeterminación, mediante la creación de nuevas estructuras democráticas transnacionales.

En mi opinión, el ideal cosmopolita corresponde a una perspectiva moral y no meramente institucional. Un elemento central del cosmopolitismo como ideal moral, que ya encontramos en los primeros que se denominaron cosmopolitas, los estoicos, es que primeramente somos seres humanos viviendo en un mundo de seres humanos y sólo incidentalmente somos miembros de comunidades políticas particulares. Desde esta perspectiva, las acciones e instituciones deben ser examinadas en relación a su contribución a la miseria humana. Para esto se asumen consideraciones más bien abstractas (cf. O'Neill, 1996, p. 56) acerca de las capacidades comunes y las formas rutinarias de vulnerabilidad que se puede asumir que todos comparten. Contrariamente al punto de vista institucional, no se trata de superar la creciente incapacidad de acción del estado, para así seguir persiguiendo las metas tradicionales bajo otras condiciones externas. Desde una perspectiva cosmopolita moral afirmamos que tenemos derechos, obligaciones y responsabilidades, que son universales.

Concepciones morales cosmopolitas usualmente apuntan a la brecha que hay en las teorías liberales entre una justificación universal y una implementación doméstica de principios de justicia. El liberalismo aspira a justificar principios de justicia para organizar una sociedad estatalmente estructurada, pero las teorías liberales van de un modo arbitrario desde la investigación acerca la organización de un estado singular hacia un mundo con muchos estados. Y donde hay estados, hay también fronteras. Pero el liberalismo no dispone de ninguna teoría sobre la legitimidad normativa de las fronteras (O`Neill, 2000, cap. 9). La pregunta acerca de la legitimidad normativa de las fronteras surge en discusiones posteriores, ejemplarmente las relativas a la secesión. Pero ella debiese estar al comienzo de la investigación normativa. 
Desde una perspectiva moral cosmopolita podemos afirmar que (a) tenemos ciertas obligaciones universales que se extienden también hacia aquellos que están fuera de las fronteras de nuestro estado de pertenencia, y que (b) estas obligaciones no se dejan reducir a obligaciones especiales. No es casual, que una aspiración cosmopolita central se puede expresar mediante una concepción de derechos humanos, cuya validez universal es corrientemente afirmada: ella se extiende por sobre las fronteras jurídicas de un estado particular, y no es reducibles a obligaciones especiales. Una implicación de este entendimiento (que un defensor del cosmopolitismo no puede rechazar) es que la protección de estos derechos debe ser garantizada, aunque esta garantía en determinados casos implique la necesidad de superar los límites del estado en dirección a estructuras internacionales o supranacionales. Diversos conflictos en la actualidad evidencian cuán difícil es garantizar la aspiración de ser gobernados por sistemas democráticos respetuosos de los derechos humanos. Por lo tanto, requeriríamos de nuevas formas de ciudadanía para asegurar la garantía de esos derechos, y así cumplir nuestras obligaciones, al menos parcialmente, más allá de las fronteras del estado bajo cuya jurisdicción vivimos. En la próxima sección, examino cinco dimensiones de ciudadanía cosmopolita relacionadas con este ideal moral.

\section{Concepciones de ciudadanía cosmopolita}

A continuación presento cinco dimensiones constitutivas de la ciudadanía cosmopolita, e indago los argumentos corrientes que se esgrimen a su favor. Por cierto, la lista no es exhaustiva. ${ }^{1}$

a) En la literatura se discute sobre un derecho democrático cosmopolita ("cosmopolitan democratic law", cf. Held, 1995). Este derecho implica: (1) un marco legal dentro del cual se reglan los conflictos entre estados, y (2) dentro del cual los individuos, cuyos derechos sean violados por parte de estados, puedan apelar. Este es un tipo de derecho internacional vinculante, que se garantiza mediante cortes de justicia internacionales, como el modelo de la Corte de Justicia Europea. Me concentraré en este último punto.

La idea de que los individuos como tales puedan hacer valer leyes internacionales contra su propio gobierno nos lleva a la idea de un estatus jurídico que nos protege contra el arbitrio de los gobernantes. Este es un

\footnotetext{
${ }^{1}$ Una concepción importante del derecho cosmopolita - a la que no refiero en este texto - refiere a sus consecuencias en la movilidad humana y se expresa como un derecho a movilidad sin fronteras (cf. entre otros Carens, 1987; 1992; Benhabib, 2004; Loewe, 2007; 2010a; 2010b; 2012; Velasco 2010; 2012).
} 
elemento constitutivo central de la ciudadanía que se encuentra en las raíces romanas de su desarrollo histórico. Es así como Paulo ejerce su derecho de ciudadano romano frente a la amenaza de la pena de muerte en un escrito al Cesar. Este estatus se define mediante derechos: individuos tendrían derechos frente a estructuras supranacionales que pueden hacer valer contra su propio gobierno. Queda abierta la pregunta, si este estatus también incluye obligaciones frente a estas estructuras o sólo frente a los estados correspondientes.

Siguiendo esta concepción, los individuos no disponen de ningún poder directo de determinación en la formación de la comunidad legal, esto es, el carácter de autodeterminación presente en los orígenes griegos de la ciudadanía. No hay ningún estatus activo. Se trata de una delgada concepción de ciudadanía cosmopolita: la competencia para hacer valer a un nivel supranacional los derechos violados por el propio gobierno. Que sea "delgada" no implica que su realización tenga consecuencias insignificantes. Por el contrario, sería un paso muy significativo. La protección y obediencia del derecho corresponden a una pasiva, pero decisiva concepción de ciudadanía (Heater, 1999, p. 143; Macedo, 1990). La ciudadanía cosmopolita estaría constituida mediante derechos humanos individualmente reclamables contra el propio gobierno. También sería posible desarrollar una protección de minorías demandable en forma colectiva - un desarrollo que se puede observar en la ONU y que en la tradición europea tiene antepasados en el modelo de protección de minorías de la Liga de las Naciones. Frente a gobiernos no democráticos que no respetan los derechos humanos, se obtienen así mecanismos de presión para implementar políticas que los garanticen.

b) Una segunda y muy discutida dimensión, se caracteriza por las demandas de justicia distributiva global que implicaría el estatus de ciudadano del mundo. Por cierto, estas demandas se expresan en diferentes marcos argumentativos, y tienen alcances disímiles. En un extremo, hay teorías maximalistas que aspiran a justificar e implementar a nivel global principios igualitarios de justicia. Buenos ejemplos son las estrategias para expandir el alcance del Principio de la Diferencia rawlsiano al contexto global (Pogge, 1989), o establecer principios distributivos que neutralicen la mala suerte de nacer en un estado nacional pobre y por tanto con posibilidades limitadas, para así alcanzar o al menos acercarnos a la realización de la igualdad global de oportunidades (Caney, 2001; Tan, 2012). En el otro extremo, hay teorías de tipo suficientaristas que aspiran a garantizar un mínimo. Esta es, por ejemplo, la aspiración de Nussbaum (2006) al establecer como exigencia de justicia la garantía de un mínimo de capacidades necesarias para poder 
desarrollar una vida con dignidad humana, o la aspiración de Pogge (2002; 2007) al abogar por un derecho humano contra la pobreza extrema, o la de Shue (1996) al argumentar a favor de un derecho a subsistencia. También es la aspiración de nuevas teorías acerca de la propiedad común de la tierra, según las cuales ella implica un derecho que se extiende hasta el presente para acceder a los recursos naturales aun no utilizados, en caso que sea necesario para satisfacer necesidades básicas (Risse, 2012). Por cierto, los marcos argumentativos también son variados. Ellos van desde extensiones del contractualismo rawlsiano (Pogge, 1989; Beitz, 1999), pasando por teorías de corte utilitaristas (Singer, 1972; 2002; Jones, 1999), hasta revitalizaciones de teorías de derecho natural (Nussbaum, 2006; Risse 2012).

Una intuición general corriente a la base de este tipo de argumentaciones es la que subyace al así llamado igualitarismo de la suerte. El igualitarismo de la suerte da una respuesta específica a la pregunta de por qué la igualdad material es relevante. Esta respuesta se basa en una intuición sobre el igual valor de los individuos como agentes morales, y así en una distinción entre suerte y agencia ( $\mathrm{o}$, entre circunstancias y decisiones): en base a una interpretación de la igualdad moral de los individuos se asume como punto de partida igualdad en la distribución de algún conjunto de bienes, y en base a una interpretación del valor moral de la agencia se considera que toda desviación desde esta debe ser justificada. Así, una distribución desigual es legítima sólo si puede ser retrotraída a la agencia de los individuos, mientras que desviaciones que se retrotraigan a la suerte (no asumida mediante agencia) son ilegítimas. Esto implica que la justicia igualitaria debe aspirar a neutralizar los efectos de circunstancias inmerecidas en la vida de los individuos. La posibilidad de realizar el propio plan de vida no debiese depender de la lotería natural y social. Según Rawls, se trata de "accidentes" y "contingencias" no merecidas. Lo que se sigue es que diferencias en distribución no merecidas (basadas en la suerte) deben ser neutralizadas mediante la justicia, mientras que diferencias en distribución basadas en elección son legítimas. ${ }^{2}$ La extensión globalista de esta intuición es que la mala fortuna se extiende a contingencias como la riqueza de la nación en que nacemos, una contingencia que tiene consecuencias profundas en la posibilidad de los individuos de realizar sus planes de vida. Este tipo de extensiones, que Rawls (1999) mismo rechaza, lleva a defender algún tipo de principio de justicia distributiva global y/o algún principio de movilidad libre a través de fronteras abiertas (Loewe, 2007; 2012), ya sea entre estados o individuos.

\footnotetext{
${ }^{2}$ Hay muchos modos de entender el contraste entre suerte y agencia y, por lo tanto, hay muchos modos de trazar la frontera entre ellos (cf. Arneson, 1989; 2000; Cohen, 1989; Dworkin, 2003).
} 
c) Una tercera dimensión de la ciudadanía cosmopolita se basa en la idea de que las decisiones deben ser tomadas en circunscripciones territoriales variables. Estas circunscripciones son definidas de tal modo que se traslapen en cada caso con todos aquellos que pueden decidir de manera legítima (Held, 1995; Pogge, 1992; cf. Beitz, 1999). Y se suele considerar que ellos son los que en cada caso se ven directamente afectados por esas decisiones. Podemos pensarlo como una estructura de círculos concéntricos. Held (1995, p. 273) piensa en referendos que cruzan a través de naciones y estados nacionales de acuerdo a la naturaleza y extensión del problema discutido. Esa idea puede ser evidenciada mediante externalidades. La idea es atractiva. El modelo parece ser productivo en cuanto a la estabilidad y al manejo de conflictos.

Considere una ilustración metafórica de las ventajas de este tipo de sistemas (cf. Bauböck, 1992): durante mucho tiempo en la producción de parabrisas se tenía el problema de que al impacto de un cuerpo extraño el cristal se quebraba en grandes trozos que podían herir a los viajeros. La solución fue la construcción de una red fina e invisible por dentro del parabrisas. Así, frente al impacto de un cuerpo, la fuerza se reparte a todo el cristal. La red se hace visible como un mosaico, pero cuanto más estrecha sea la red, menor será el peligro de que el objeto lo atraviese. Esa sería la ventaja si existiese un sistema de referendos como el descrito: los diferentes círculos de toma de decisiones se traslaparían, de modo que ningún impacto las destruiría con consecuencias peligrosas. Desde la perspectiva de las dimensiones constitutivas, mediante los referendos se incluye el momento activo de la ciudadanía en la concepción cosmopolita.

Sin embargo, hay buenas razones para ser escéptico con respecto a estas posiciones. En política, al menos como la conocemos hasta hoy, la determinación de las circunscripciones relevantes es central. ¿Cómo podemos aquí determinar las circunscripciones relevantes? ¿Quiénes son los afectados relevantes en un caso de contaminación medioambiental? ¿Cómo podemos determinar que externalidades son aun relevantes? Y en un problema de autodeterminación y secesión ¿quiénes son, por ejemplo, los afectados relevantes en caso de una posible secesión del País Vasco? ¿Son todos los españoles o sólo los vascos? El problema no es que no podamos determinar las circunscripciones (a pesar de que es una tarea difícil). El problema es que toda la política estaría ocupada con el problema de fijar las circunscripciones relevantes. Porque hay diferentes intereses y la determinación de las circunscripciones es central para obtener los resultados previstos, cada grupo de interés va a argumentar para considerar los criterios particulares para 
determinar a los afectados que más los favorezcan. Y esto con una lógica casuística. Pero una concepción casuística de la política no provee estabilidad (cf. Miller, 1999). La provisión de estabilidad está mejor servida mediante constituciones relativamente estables, que hacen obligantes diferentes reglas en diferentes contextos antes del acontecer de los casos particulares. Con una constitución podemos estipular quién es competente en qué decisiones, qué puede ser decidido y qué no, cuándo debe ser algo decidido y cuándo no, hasta cuándo puede ser decidido y a partir de cuándo etc. (cf. Elster, 2000). En un sistema federativo se fijan desde un comienzo las correspondientes competencias: que decide el Land o Regiones, Cantones etc., que el Bund o la federación. Si tenemos que revisar continuamente las competencias frente a cada problema y cada afectado, no quedaría ningún parabrisas. Y hay una buena razón porque competencias fijas proveen estabilidad: no se sabe de antemano quién gana y quién pierde. Contrariamente a esto, la concepción casuística de las competencias plantea antes de cada o de muchas decisiones la pregunta acerca de quiénes serán los ganadores y perdedores. El problema permanece en tanto no haya una autoridad internacional que fije cada competencia para cada caso posible antes de que acontezca.

d) Una cuarta dimensión de la ciudadanía cosmopolita se encuentra en la idea de una sociedad civil global ("global civil society" u "Öffentlichkeit"), esto es, la participación de individuos (ciudadanos del mundo) en grupos con intereses particulares transnacionales. Falk (1995, p. 211-212; 2009) los denomina ciudadanos peregrinos ("citizen pilgrims"). La metáfora del ciudadano peregrino describe

the spirit of a sojourner, committed to transformation that is spiritual as well as material, that is premised on the wholeness and equality of the human family and that is not disposed to put much trust in prospects for a technical fix that might claim to enable global governance to succeed provided it is self-delineated as a functional project and nothing more (Falk, 2009, p. 78).

La participación y apoyo de grupos medioambientalistas, de protección de los derechos humanos, organizaciones de ayuda humanitaria etc. son actividades ampliamente difundidas. Hay al menos tres posibilidades: (1) participación en organizaciones de la sociedad civil con un alcance global; (2) participación en instituciones políticas supranacionales ya existentes; (3) defensa de nuevas posibilidades para fortalecer de la ciudadanía mundial política (cf. Heater, 1999). 
e) En los debates acerca del cosmopolitismo la educación cosmopolita ocupa un lugar destacado. Si bien en sentido estricto ésta se relaciona de diferentes modos con las cuatro dimensiones recién descritas, la importancia que se le otorga torna razonable considerarla como una dimensión propia. Marta Nussbaum sostiene que el cosmopolitismo tiene sobre todo consecuencias importantes al nivel de la educación (Nussbaum, 1996; 1997). Niños deberían aprender en la escuela sobre tradiciones y culturas, también sobre las de aquellos que están presentes en la sala de clases, pero no, y esto crucial, para que aquellos sobre cuya cultura y tradiciones se aprende, alcancen un mayor auto-respeto - como sostienen, por ejemplo, algunas posiciones multiculturales (Kymlicka, 1995). Ese no puede ser el objetivo de la educación cosmopolita. Si el objetivo de la enseñanza es aprender algo sobre la propia cultura para alcanzar un mayor auto-respeto, desistimos del cosmopolitismo. Se aprende sobre tradiciones y culturas, propias y ajenas, y desde la niñez temprana, mediante cuentos e historias de otras regiones, para ver y para aprender cuanto de común hay en diferentes culturas y tradiciones, para que así lo ajeno deje de serlo tanto. Es como en la buena literatura: se descubre lo universal en lo particular. Se aprende que los problemas de los hombres en el mundo son parecidos. Que los problemas de personas de culturas ajenas y de otros países no son los problemas de esas personas en particular, porque ellos son como son (o porque no son como nosotros), sino que son los problemas de cada persona que se encuentre en tales circunstancias. Y que nuestros problemas son problemas humanos, que surgen en circunstancias específicas.

\section{Obligaciones hacia nuestros compatriotas}

Si se acepta el marco teórico conceptual ofrecido por una concepción unitaria paradigmática de ciudadanía, es común afirmar que la ciudadanía sólo es posible dentro de estados particulares, usualmente entendidos como estados nacionales. Desde esta perspectiva, es corriente criticar al cosmopolitismo porque debido a su racionalidad abstracta y a sus altas exigencias no sería capaz de generar (1) ni una descripción fenomenológica plausible de nuestro mundo moral (2) ni una organización institucional factible (cf. Nussbaum, 1996). ${ }^{3}$ El ciudadano de la cosmopolis sería un "ciudadano de ningún lugar" (MacIntyre, 1995).

\footnotetext{
${ }^{3}$ Otras críticas afirman que: (a) esta forma de ciudadanía destruye las tradiciones locales y nacionales, y de este modo aspira a crear o efectivamente crea homogeneidad cultural; (b) La idea de una ciudadanía cosmopolita se puede equiparar con imperialismo cultural, ya que los valores, que estarían a la base de ésta no son (1) valores compartidos por todos, o son (2) valores exclusivamente occidentales; (c) Una ciudadanía del mundo no deja espacio libre para las lealtades particulares; (d) Habría una relación necesaria entre ciudadanía y estado nacional,
} 
Como alternativa a las supuestas deficiencias que evidenciarían estas críticas, se suele argumentar a favor de teorías liberales nacionalistas o teorías estatistas. De acuerdo a las primeras -y de un modo similar al nacionalismo romántico de cuño herderiano-, los individuos sólo pueden desarrollar una vida lograda dentro de una cultura nacional, ya sea aquella en la que nacen o en la que crecen y socializan (cf. Tamir, 1993; Kymlicka, 1995; Miller 1995; Calsamiglia, 2000). Una nacionalidad compartida constituiría -en base a razones diferentes- un vínculo normativo elemental (cf. Loewe, 2007a). Siguiendo a David Miller (1995), quien articula una de las teorías liberales nacionalistas más discutidas en el debate, naciones son "comunidades éticas". Las teorías estatistas, por su parte, expresan una posición muy difundida en los contextos internacionales, a cuyo favor es posible argüir argumentos muy convincentes: la organización actual del mundo entre estados representa el punto de vista natural para nuestros argumentos morales en relación a los derechos y deberes de las personas, porque es dentro de las sociedades estatalmente organizadas que se dan las condiciones que permiten razonablemente estipular obligaciones de justicia (por ejemplo, la cooperación: Rawls, 1971; o coacción: Nagel, 2005). Sin embargo, las críticas mencionadas se basan en malentendidos.

Primero: el núcleo moral del cosmopolitismo es también el del liberalismo: el individualismo, el universalismo, y el igualitarismo. Individualismo, porque el individuo es la última unidad con un valor moral (o porque es la última fuente para determinar los valores morales); universalismo, porque todos los individuos cuentan; e igualitarismo, porque todos cuentan por igual. Pero este núcleo no implica que tengamos exactamente las mismas obligaciones hacia todos los otros. Dentro de una posición cosmopolita hay suficiente espacio para incluir obligaciones especiales hacia nuestros conciudadanos. Esto es válido también en el caso de obligaciones especiales que surgen en el contexto de relaciones particulares tanto en grupos de filiación como en el contexto de relaciones íntimas (como la familia): ser cosmopolita no implica que no podamos privilegiar (en ciertas circunstancias) a aquellos con los que nos sentimos más estrechamente vinculados por sobre extraños. Las distinciones usuales entre las obligaciones hacia nuestros conciudadanos y hacia aquellos que no lo son, entre las obligaciones hacia aquellos que están bajo la jurisdicción de un estado sin ser ciudadanos de éste (ciudadanos de otros estados o apátridas), y hacia aquellos que no están bajo la jurisdicción de

que tornaría imposible ir más allá de sus estructuras institucionales. Correspondientemente, el discurso acerca de una "ciudadanía del mundo" sería (a) contradictorio, (b) o tan falto de contenido, que referiría a algo diferente a aquello que se puede entender como ciudadanía. Si bien considero que hay buenos argumentos, no me referiré a ellas. 
este estado etc., no se oponen en principio al cosmopolitismo. De este modo, sí es compatible con una descripción fenomenológica de nuestro mundo moral (Loewe, 2010b).

Segundo: la aceptación de una posición cosmopolita no implica abogar por el fin de las fronteras políticas, lo que podría llevar a una organización deficiente, y tampoco implica que haya que apoyar el surgimiento de determinadas instituciones que haya buenas razones para rechazar. Al entendernos como ciudadanos del mundo no estamos obligados a apoyar la creación de un estado mundial (contra el cual es posible articular argumentos convincentes). A diferencia de la perspectiva institucional empírica, la perspectiva moral cosmopolita no dice que la ciudadanía precisa en la actualidad de nuevas instituciones. Lo que ella dice es que tenemos determinadas obligaciones internacionales, que se pueden cumplir a través de nuevas instituciones. Podemos aceptar una posición cosmopolita y a pesar de esto, o quizás debido a esto, sostener que la mejor organización política del mundo es la que se constituye mediante estados. Pero a diferencia del estatismo y del nacionalismo liberal, el cosmopolitismo que reconoce obligaciones especiales hacia los miembros de nuestra comunidad política, no tiene una posición de principio con respecto a la pregunta acerca de la legitimidad de las fronteras vigentes. Si el trazar nuevas fronteras tornase más sencillo el mantenimiento o la creación de instituciones cosmopolitas, y estas nuevas fronteras fuesen deseadas por todos aquellos en el área, el cosmopolitismo tiene poco que oponer. Correspondientemente, si la traza de nuevas fronteras hace difícil el mantenimiento o buen funcionamiento de instituciones liberales, el cosmopolitismo tiene un buen caso en su contra (cf. Buchanan, 1991).

Lo que distingue estructuralmente al cosmopolitismo moral del nacionalismo liberal y del estatismo, no es que los partidarios del cosmopolitismo piensen que ese tipo de diferenciaciones en los derechos no sea legítima, mientras que los partidarios del nacionalismo liberal o del estatismo las consideren legítimas. La diferencia recae en la dirección en la justificación de esos paquetes diferenciados de derechos y deberes.

Liberales nacionalistas y estatistas consideran que primero están las obligaciones hacia los connacionales -ya sea porque naciones son comunidades éticas, como afirma Miller (1995); o porque los estados son los lugares en los que se puede dar la cooperación o coacción que torna razonable hablar de obligaciones de justicia, como afirman autores estatistas (Nagel, 2005). De modo ejemplar podemos observar esta estructura en $A$ theory of justice y en The law of peoples de John Rawls (Loewe, 2015). Al tomar como punto de partida de su razonamiento normativo el paradigma de un mundo dividido 
entre naciones o estados, tratan de justificar que en cuanto ciudadanos de un estado o miembros de una nación, tenemos un determinado paquete de derechos y deberes, y sólo entonces, en un segundo paso, se preguntan si hay obligaciones hacia aquellos que no están bajo la jurisdicción de este estado o que no pertenecen a la nación, y en caso que las hubiese, cuáles serían. Por el contrario, cosmopolitas consideran que tenemos obligaciones universales hacia todos los seres humanos, y especiales hacia nuestros conciudadanos. El cumplimiento de las segundas está supeditada al cumplimiento de las primeras (como sea que se caracterice su materialidad). Así, primero tenemos que determinar qué derechos tienen los individuos en cuantos seres humanos con independencia de la correspondiente membresía estatal o nacional, y entonces, en un segundo paso, tenemos que argumentar por la legitimidad de diferentes paquetes de derechos y obligaciones en caso de ciudadanos o nacionales y no-ciudadanos o no-nacionales. Antes de desarrollar la argumentación, no podemos saber si los paquetes de derechos y obligaciones que resulten se diferenciaran en razón de la dirección en la argumentación.

\section{El ciudadano cosmopolita}

Ser cosmopolita no significa sentirse en casa en muchos países, comer comidas exóticas o ser políglota. A un nivel basal, ser cosmopolita es una manera de pensar, y puede ser una disposición de vida (Waldron, 1995). Recelar del ciudadano cosmopolita y sus lealtades, y por tanto proponer otorgar centralidad en la vida política al sentimiento del orgullo nacional y de una identidad nacional compartida (Rorty, 1994), o dar - como en los tiempos de la construcción de la nación - más espacio a la bandera y a otros símbolos nacionales (Fletcher, 1993) o proponer un "panteón de héroes" a ser honrado (Galston, 1991) es completamente injustificado. Sólo puede parecer justificado si se sostiene que la identidad y las lealtades se deben restringir a un grupo particular, que normalmente se identifica con la nación o el de los ciudadanos. La ciudadanía cosmopolita implica el traspaso de lealtades fuera de las fronteras políticas y debido a razones universales, pero no se deja atrapar con los conceptos dualísticos de "lo uno o lo otro". Un ciudadano cosmopolita se deja entender mejor con los conceptos de "tanto lo uno, como lo otro". Así, se puede estar orgulloso de lo mejor de las tradiciones propias (lo que no quiere decir no ser crítico con respecto a ellas, sino lo contrario) y al mismo tiempo se puede pensar y actuar de modo cosmopolita. El cosmopolitismo deja suficiente espacio para fundar obligaciones especiales hacia a nuestros conciudadanos.

Que la ciudadanía cosmopolita tenga consecuencias al nivel de las actitudes y de las acciones personales, no implica necesariamente que tenga 
que ver sólo con el cosmos pero no con la polis. Como examiné, asumir una ciudadanía cosmopolita tiene consecuencias a muchos niveles entre y dentro de las sociedades y de diversas estructuras internacionales y supranacionales. Ejemplos claros son el avance en la protección de derechos humanos (comentado en la primera dimensión), el medioambiente y en general en la producción de bienes públicos mundiales. Tomar en serio que no todo el mundo puede alcanzar los estándares de vida de los países ricos, porque el medioambiente no lo soportaría (Nussbaum, 1996, p. 12), debe hacernos reflexionar. En este sentido la ciudadanía cosmopolita no es ni incoherente ni demasiado delgada. Además, desde el cosmopolitismo se puede articular buenos argumentos a favor de la justicia distributiva global (segunda dimensión). Resta un largo e incierto camino hacia la implementación de mecanismos institucionales de justicia distributiva entre sociedades. Pero es una tarea de ciudadanos cosmopolitas ser socialmente activos en este proceso (cuarta dimensión). Esto tiene implicancias importantes al nivel de la educación (quinta dimensión). Hace pocos siglos parecía absurdo para la mayoría de las personas la idea de que las sociedades no tuviesen formas de esclavitud. Nadie defiende hoy seriamente la esclavitud (aunque sí, hasta hace poco), aunque ciertamente esclavitud y formas similares de dominación son corrientes. El editor de John Stuart Mill se quejaba de que The subjection of women fue el único libro de Mill con cuya publicación perdió dinero. Sus tesis acerca de la igualdad legal de hombres y mujeres encuentran hoy en día un amplio apoyo - aunque no necesariamente entre feministas, y por buenas razones. Como afirma Barry (1999), no es descabellado suponer o aventurar que en un futuro próximo, se va a mirar hacia nuestro tiempo y se va a estar sorprendido de que una transferencia de sólo un 0.2 o 0.3 del Producto Interno Bruto de países ricos a pobres se consideraba como normativamente apropiada.

\section{Referências}

ARNESON, Richard. Equality and equal opportunity for welfare. Philosophical Studies, v. 56, n. 1, p. 77-93, $1989<10.1007 /$ BF00646210>.

ARNESON, Richard. Luck egalitarianism and prioritarism, Ethics, v. 110, n. 2, p. 339$49,2000<10.1086 / 233272>$.

BARRY, Brian. A liberal theory of justice. Oxford: Oxford University Press, 1974.

BARRY, Brian. Statism and nationalism: a cosmopolitan critique. In: Ian Shapiro; Lea Brilmayer (org.). Global justice. New York: New York University Press, 1999. p. 12-66.

BAUBÖCK, Rainer. Zur Zukunft des Nationalismus in Europa. Unvollendeter Nationenbau oder postnationale Gesellschaft? Wissenschaft \& Frieden: Facetten der Gewalt, v. 4, texto 960, 1992. <http://www.wissenschaft-und-frieden.de/seite. php?artikelID $=0960>$ [Friedensbericht 1991, Wien, 1992, p. 159-181]. 
BAUBÖCK, Rainer. Transnational citizenship: membership and rights in international migration. Aldershot: Edward Elgar, 1994.

BECK, Ulrich. Was ist Globalisierung. Frankfurt am Main: Suhrkamp, 1997.

BEITZ, Charles. Political theory and international relations. Princeton: Princeton University Press, 1999.

BENHABIB, Seyla. The rights of others. Cambridge: Cambridge University Press, 2004.

BUCHANAN, Allen. Secession: the morality of political divorce from Fort Sumter to Lithuania and Quebec. Boulder: Westview Press, 1991.

CALSAMIGLIA, Albert. Cuestiones de lealtad: límites del liberalismo. Barcelona: Paidós, 2000.

CANEY, Simon. Cosmopolitan justice and equalizing opportunities. Metaphilosophy, v. 32, n. 1-2, p. 113-134, $2001<10.1111 / 1467-9973.00178>$.

CANEY, Simon. Justice beyond borders: a global political theory. Oxford: Oxford University Press, $2005<10.1093 / 019829350 X .001 .0001>$.

CANEY, Simon. Global poverty and human rights: the case for positive duties. In: Thomas Pogge (org.). Freedom from poverty as a human right. Oxford: Oxford University Press, 2007. p. 275-302.

CARENS, Joseph. Aliens and citizens: the case of open borders. The Review of Politics, v. 49, n. 2, p. 251-273, $1987<10.1017 /$ S0034670500033817>.

CARENS, Joseph. Migration and morality: a liberal egalitarian perspective. In: Brian Barry; Robert Gooding (orgs.). Free movement. University Park: Penn State University Press, 1992. p .25-47.

COHEN, G. A. On the currency of egalitarian justice. Ethics, v. 99, n. 4, p. 906-944, $1989<10.1086 / 293126>$.

DAGGER, Richard. Civic virtues: rights, citizenship, and Republican liberalism. Oxford: Oxford University Press, 1997.

DWORKIN, Ronald. Equality, luck, and hierarchy. Philosophy \& Public Affairs, v. 31, n. $2,190-198,2003<10.1111 / j .1088-4963.2003 .00190 . x>$.

ELSTER, Jon. Ulysses unbound: studies in rationality, precommitment, and restraints. Cambridge: Cambridge University Press, $2000<10.1017 /$ CBO9780511625008>.

FABRE, Cécile. Global distributive justice: an egalitarian perspective. Canadian Journal of Philosophy, v. 35 (sup. 1), p. 139-164, 2005.

FALK, Richard. On human governance: towards a new global politics. Cambridge: Polity Press, 1995.

FALK, Richard. Achieving human rights. New York: Routledge, 2009.

FLETCHER, George. Loyalty: an essay on the morality of relationships. Oxford: Oxford University Press, 1993.

GALSTON, William. Liberal purposes: goods, virtues and diversity in the liberal state. Cambridge: Cambridge University Press, $1991<10.1017 /$ CBO9781139172462>. 
HABERMAS, Jürgen. Faktizität und Geltung: Beiträge zur Diskurstheorie des Rechts und des demokratischen Rechtsstaates. Frankfurt am Main: Suhrkamp, 1992.

HEATER, D. B. Citizenship: the civic ideal in world history, politics, and education. London: Longman Group, 1999.

HELD, David. Democracy and the global order: from the modern State to cosmopolitan Governance. Cambridge: Polity Press, 1995.

HÖFFE, Otfried. Demokratie im Zeitalter der Globalisierung. München: C. H. Beck, 1999.

HÖFFE, Otfried. Wirtschaftsbürger, Staatsbürger, Weltbürger. München: C. H. Beck, 2004.

HUTCHINGS, Kimberly; DANNREUTHER, R. Cosmopolitan citizenship. New York: St. Martin's Press, 1999.

JONES, Charles. Global justice: defending cosmopolitanism. Oxford: Oxford University Press, 1999.

KYMLICKA, Will. Multicultural citizenship. Oxford: Oxford University Press, 1995.

KYMLICKA, Will; NORMAN, Wayne. Return of the citizen: a survey of recent work on citizenship theory. Ethics, v. 104, n. 2, p. 352-381, $1994<10.1086 / 293605>$.

LOEWE, Daniel. Inmigración y el derecho de gentes de John Rawls. Revista de Ciencia Política, v. 27, n. 2, p. 23-48, 2007.

LOEWE, Daniel. Teorías de justicia igualitaria y derechos culturales diferenciados. Isegoría, n. 36, p. 275-302, 2007a <10.3989/isegoria.2007.i36.69>.

LOEWE, Daniel. La justicia de la inmigración. Civitas, v. 9, n. 3, p. 496-513, 2009 $<10.15448 / 1984-7289.2009 .3 .6904>$.

LOEWE, Daniel. Los náufragos de nuestro mundo: el caso de los refugiados. Arbor, v. 186 , n. 744, p. 555-570, 2010a <10.3989/arbor.2010.744n1217>.

LOEWE, Daniel. Liberalismo, inmigración y justicia global. Isegoría, n. 43, p. 435-458, 2010 b $<10.3989$ /isegoria.2010.i43.702>.

LOEWE, Daniel. Obligaciones de justicia: open borders o justicia distributiva? Arbor, v. 188 , n. 755 , p. $475-88,2012<10.3989$ /arbor.2012.755n3002>.

LOEWE, Daniel. ¿Cuán liberal es la teoría de las relaciones internacionales de Rawls? Veritas, v. 60, n. 1, p. e1-e35, $2015<10.15448 / 1984-6746.2015 .1 .17988>$.

MACEDO, Stephen. Liberal virtues: citizenship, virtue and community in liberal constitutionalism. Oxford: Clarendon Press, 1990.

MacINTYRE, Alasdair. Is patriotism a virtue? In: Ronald Beiner (org.). Theorizing citizenship. Albany: State University of New York Press, 1995. p. 209-228.

MILLER, David. On nationality. Oxford: Oxford University Press, 1995.

MILLER, David. Justice and global inequality. In: Andrew Hurrell; Ngaire N. Woods (orgs.). Inequality, globalization, and world politics. Oxford: Oxford University Press, $1999<10.1093 / 0198295669.003 .0008>$. 
MOELLENDORF, Darrel. Cosmopolitan justice. Boulder: Westview Press, 2002.

NAGEL, Thomas. The problem of global justice. Philosophy \& Public Affairs, v. 33, n. 2, p. 113-147, $2005<10.1111 /$ j.1088-4963.2005.00027.x>.

NUSSBAUM, Martha. Patriotism and cosmopolitism. In: Joshua Cohen (org.). For love of country: debating the limits of patriotism. Boston: Beacon Press, 1996.

NUSSBAUM, Martha. Cultivating humanity: a classical defense of reform in liberal education. Oxford: Oxford University Press, 1997.

NUSSBAUM, Martha. Frontiers of justice. Harvard University Press, 2006.

O `NEILL, Onora. Towards justice and virtue. Cambridge: Cambridge University Press, 1996.

O`NEILL, Onora. Bounds of justice. Cambridge: Cambridge University Press, 2000.

POGGE, Thomas. Realizing Rawls. Ithaca: Cornell University Press, 1989.

POGGE, Thomas. Cosmopolitanism and sovereignty. Ethics, v. 103, n. 1, p. 48-75, $1992<10.1086 / 293470>$.

POGGE, Thomas. World poverty and human rights. Cambridge: Polity Press, 2002.

POGGE, Thomas. Severe poverty as a human rights violation. In: Thomas Pogge (org.). Freedom from poverty as a human right. Oxford: Oxford University Press, 2007.

RAWLS, John. A theory of justice. Harvard University Press, 1971.

RAWLS, John. The law of peoples. In: Stephen Shute; Susan Hurley (orgs.). On human rights. New York: Basic Books, 1993.

RAWLS, John. The law of peoples with the idea of public reason revisited. Harvard University Press, 1999.

RISSE, Mathias. On global justice. Princeton: Princeton University Press, 2012.

RORTY, Richard. New York Times, 13 Feb., 1994.

SHUE, Henry. Basic rights. Princeton: Princeton University Press, 1996.

SINGER, Peter. Famine, affluence and morality. Philosophy and Public Affairs. n. 1, n. 3, p. 229-243, 1972.

SINGER, Peter. Outsiders: our obligations to those beyond our borders. In: Deen K. Chatterjee (org.). The ethics of assistance. Cambridge: Cambridge University Press, 2004. p. 11-32.

SINGER, Peter. One world. New Haven: Yale University Press, 2002.

SOYSAL, Yasemin Nuhoglu. Changing parameters of citizenship and claims-making: organized Islam in European Public Spheres. Theory and Society. v. 26, n. 4, p. 509-527, $1997<10.1023 / \mathrm{A}: 1006886630358>$.

SPINNER-HALEV, Jeff. The boundaries of citizenship: race, ethnicity and nationality in the liberal state. Baltimore: Johns Hopkins University Press, 1994.

TAMIR, Yael. Liberal nationalism. Princeton: Princeton University Press, 1993.

TAN, Kok-Chor. Toleration, diversity, and global justice. University Park, Pennsylvania: Penn. State University Press, 2000. 
TAN, Kok-Chor. Justice without borders. Cambridge: Cambridge University Press, 2004.

TAN, Kok-Chor. Justice, institutions, \& luck. Oxford: Oxford University Press, 2012.

TURNER, Bryan. Outline of a theory of citizenship. Sociology. v. 24, n. 2, p. 189-218, $1990<10.1177 / 0038038590024002002>$.

VELASCO, Juan Carlos. Dejando atrás las fronteras. Arbor, v. 186, n. 744, p. 585-601, $2010<10.3989$ /arbor.2010.744n1219>.

VELASCO, Juan Carlos. Movilidad humana y fronteras abiertas. Claves de razón práctica, n. 219, p. 28-35, 2012.

WALDRON, Jeremy. Minority cultures and the cosmopolitan alternative. In: Will Kymlicka (org.). The rights of minority cultures. Oxford: Oxford University Press, 1995. p. 93-119.

WIENER, Antje. Making sense of the new geography of citizenship: fragmented citizenship in the European Union. Theory and Society, v. 26, n. 4, p. 529-660, 1997 $<10.1023 / \mathrm{A}: 1006809913519>$.

YOUNG, Iris Marion. Justice and the politics of difference. Princeton: Princeton University Press, 1990.

Recebido em: 2 nov. 2015

Aprovado em: 1ํ dez. 2015

Autor correspondente

Daniel Loewe (a-229)

Escuela de gobierno

Av. Diagonal las torres 2640 - Peñalolen

Santiago, Chile 